\title{
Mapeamento Sistemático sobre Sistemas de Recomendações Educacionais
}

\author{
Victor A. S. Ferreira ${ }^{1}$, Germano C. Vasconcelos ${ }^{1}$, Rozelma S. França ${ }^{1,2}$ \\ ${ }^{1}$ Centro de Informática - Universidade Federal de Pernambuco (UFPE) \\ 50.740-560 - Recife, PE - Brasil \\ ${ }^{2}$ Departamento de Educação - Universidade Federal Rural de Pernambuco (UFPE) \\ 52.171-900 - Recife, PE - Brasil \\ \{vasf, gcv, rsf2\}@cin.ufpe.br
}

\begin{abstract}
Considering the need to consider the specificities of students in their learning process, systems of educational recommendations (SRE) can be used in this process, suggesting materials that support the construction of students' knowledge, considering their interests and needs. In this context, this paper aims to identify the current state of research on SRE in Brazil, by performing a systematic mapping of literature. The research had the analysis of studies published in the last five years in the main scientific vehicles of Informatics in Education in the country: SBIE, WIE and RBIE. The reported results refer to the historical evolution of the publications about SRE in Brazil, the institutions involved in the research, the target public, recommended resources, the most used recommendation techniques, as well as the evaluation of these systems.
\end{abstract}

Resumo. Tendo em vista a necessidade de considerar as especificidades dos estudantes em seu processo de aprendizagem, sistemas de recomendações educacionais (SRE) podem ser usados nesse processo, sugerindo materiais que apoiem a construção do conhecimento dos educandos, considerando seus interesses e necessidades. Neste contexto, este trabalho visa identificar o estado atual da pesquisa sobre SRE no Brasil, pela realização de um mapeamento sistemático de literatura. A pesquisa contou com a análise de estudos publicados nos últimos cinco anos nos principais veículos científicos de Informática na Educação no país: SBIE, WIE e RBIE. Os resultados reportados dizem respeito à evolução histórico das publicações sobre SRE no Brasil, as instituições envolvidas nas pesquisas, o público-alvo, os recursos recomendados, as técnicas de recomendação mais utilizadas, como também sobre a avaliação desses sistemas.

\section{Introdução}

De acordo com o Relatório Analítico da Aprendizagem a Distância no Brasil (2015) elaborado pela Associação Brasileira de Educação a Distância (ABED), houve um aumento significativo no número de matrículas realizadas em cursos desta modalidade ofertados por diferentes categorias administrativas. Em tais cursos, tecnologias de informação e comunicação são usadas para apoiar os processos de ensino e aprendizagem, a exemplo de Ambientes Virtuais de Aprendizagem (AVAs).

Nesses cursos, ao mesmo tempo em que turmas são formadas por uma grande 
quantidade de discentes, é preciso considerar suas especificidades no processo de aprendizagem, configurando-se um desafio aos professores quando se pretende identificar e recomendar materiais personalizados para cada estudante, baseando-se em suas necessidades, interesses e competências a serem desenvolvidas (CAZELLA et al., 2009).

Nesse contexto, sistemas de recomendação (SR) podem ser usados provendo materiais didáticos que considerem as preferências dos estudantes e ajudando-os a alcançar os objetivos de aprendizagem do curso. Diferentes técnicas podem ser usadas na recomendação de recursos, como as filtragens por conteúdo, colaborativa e híbrida. Tais técnicas têm sido empregadas em aplicações de diferentes contextos, incluindo o educacional (COSTA et al., 2013).

No Brasil, diversas pesquisas têm sido realizadas sobre a temática, investigando técnicas de recomendação que melhor se adaptam às necessidades de estudantes e professores nos processos de ensino e aprendizagem, como também sua aplicação em AVAs (COSTA et al., 2013). No intuito de melhor entender esse cenário no Brasil, identificando as características dos sistemas de recomendações educacionais (SRE) propostos e usados, como também a forma como têm sido avaliados, esta pesquisa analisou a produção científica nacional sobre SRE, por meio de um mapeamento sistemático. A pesquisa contou com a análise de estudos publicados nos últimos 5 anos nos três principais veículos de publicação nacional sobre Informática na Educação (IE): o Simpósio Brasileiro de Informática na Educação (SBIE), o Workshop de Informática na Escola (WIE) e a Revista Brasileira de Informática na Educação (RBIE).

O restante do artigo está estruturado da seguinte forma: a seção 2 apresenta mapeamentos e revisões sistemáticas voltadas à análise de estudos nacionais em temas relacionados à IE e, na seção 3, o método empregado neste trabalho é descrito. Na seção 4 são exibidos os resultados obtidos a partir da análise dos 22 estudos selecionados sobre SRE. Por fim, a seção 5 traz as considerações finais acerca de todo o trabalho e apresenta alguns trabalhos futuros.

\section{Trabalhos relacionados}

Ao analisar-se a literatura, é possível identificar diversos estudos secundários que versam sobre a identificação de tendências e lacunas de pesquisas em IE no contexto brasileiro. Em Magalhães et al. (2013) o objetivo foi caracterizar a pesquisa em IE no Brasil com base nas publicações do SBIE entre os anos de 2001 e 2012. Para tanto, um mapeamento sistemático foi realizado e como resultados pode-se identificar que a pesquisa na área é diversificada em relação aos tópicos pesquisados, abrangendo diversos contextos, situações e público. Contudo, é limitada em relação aos métodos de pesquisa, apresentando um número relativamente baixo de estudos empíricos.

Já Aureliano e Tedesco (2012) estavam interessadas na análise de estudos sobre o processo de ensino-aprendizagem de programação para iniciantes publicados no SBIE e no WIE no período de 2002 a 2011. Seus resultados apontam que houve um aumento no interesse da comunidade brasileira na área, que a maioria das pesquisas está sendo realizada por pesquisadores localizados nas regiões Sul e Sudeste do Brasil, que as pesquisas focam principalmente no ensino superior e que grande parte delas apresenta novas ferramentas de software. 
Com o enfoque em SRE, Aguiar et al. (2014) mapearam iniciativas sobre o tema publicadas até 2013 em eventos e periódicos nacionais de IE. Como resultados, identificaram o crescente número de estudos publicados na área e que o Sul e o Nordeste foram as regiões de maior concentração de pesquisas. Além disso, constataram que geralmente os estudos são focados na recomendação de objetos de aprendizagem (OAs) para estudantes, considerando principalmente o conteúdo desses OAs no processo de recomendação, sendo possível identificar também aqueles que envolvem a recomendação de outros recursos, como pessoas. De modo complementar, Aguiar et al. (2015) verificaram as estratégias de avaliação adotadas pelos SRE propostos nos estudos revisados.

Já Pontes et al. (2014) realizaram uma revisão sistemática de estudos sobre filtragens de recomendação de OAs publicados nos anais do Congresso Brasileiro de Informática na Educação (CBIE) no período de 2008 a 2013. A partir da análise realizada verificou-se um crescimento nas produções científicas sobre o tema. Também, que boa parte das recomendações se utiliza da filtragem por conteúdo e que os estudos revisados reconhecem a relevância dos sistemas de colaboração, se utilizando desta estrutura de filtragem.

Após a análise da literatura, não se identificou a existência de estudos secundários que versem sobre SRE e estratégias para sua avaliação considerando os estudos publicados nos últimos 5 anos nos principais veículos nacionais de IE. Este, então, consiste no foco do mapeamento sistemático descrito neste artigo.

\section{Método}

Objetivando identificar tendências e lacunas de pesquisa sobre SRE no contexto brasileiro, um mapeamento sistemático foi realizado. Segundo Kitchenham (2007), mapeamentos sistemáticos são projetados para fornecer uma visão geral de uma área de pesquisa, fornecendo evidências sobre o tópico investigado. Para sua realização, diversas etapas são executadas e nas seções seguintes são descritas as definidas para esta pesquisa.

\subsection{Questões de pesquisa}

A questão central deste estudo é: Qual é o estado atual da pesquisa sobre SRE no Brasil? . Para responder à questão principal deste estudo foram definidas as seguintes questões específicas de pesquisa:

- QP1: Como se deu a evolução histórica das publicações sobre SRE nos principais eventos e periódico nacionais sobre IE no país?

- QP2: Quais são as instituições que mais publicaram estudos primários sobre SRE nos principais veículos nacionais de publicação científica sobre IE?

- QP3: Quais os usuários-alvo das recomendações nessas iniciativas?

- QP4: Quais os recursos educacionais recomendados nessas pesquisas?

- QP5: Quais as técnicas de recomendação mais utilizadas nos SRE?

- QP6: Como as avaliações dos sistemas de recomendação têm sido realizadas?

\subsection{Critérios de inclusão e exclusão}

Para orientar o processo de seleção dos estudos, critérios de exclusão foram definidos. 
VI Congresso Brasileiro de Informática na Educação (CBIE 2017)

Anais do XXVIII Simpósio Brasileiro de Informática na Educação (SBIE 2017)

Caso um estudo atendesse a pelo menos um dos critérios listados a seguir, ele era excluído do processo de análise:

- Artigos não acessíveis na íntegra;

- Artigos resumidos, tutoriais, relatórios de workshop;

- Estudos secundários e terciários;

- Estudos duplicados: apenas o mais atual foi incluído;

- Artigos que expressam pontos de vistas pessoais ou opiniões de especialistas;

- Artigos que não tratem sobre recomendação de recursos educacionais, ou aqueles em que esta funcionalidade não seja o enfoque, ou pelo menos parte em destaque, dos estudos selecionados;

- Estudos que tratem de SRE apenas como trabalhos futuros;

- Estudos sem avaliação dos SRE propostos ou usados;

- Estudos publicados fora do período 2012-2016.

Para ser incluído, então, o estudo deveria atender ao seguinte critério:

- Trabalhos científicos completos que tratam sobre SRE e que realizem avaliação da proposta.

\subsection{Fontes de dados, estratégia de busca e seleção dos estudos}

A busca automática foi realizada nos três principais engenhos de busca nacionais sobre Informática na Educação: SBIE (www.br-ie.org/pub/index.php/sbie), WIE (www.brie.org/pub/index.php/wie) e RBIE (www.br-ie.org/pub/index.php/rbie) . Para tal, a string recom* foi usada e, por ser abrangente, pode evitar a perda de possíveis resultados importantes.

Vale pontuar que ao realizar a busca automática nas três bases mencionadas e ler estudos retornados, foi observado que alguns desses trabalhos faziam referência a pesquisas que deveriam ter sido retornadas, mas que não foram, mesmo sendo usada uma string abrangente como a aqui proposta. Assim, para complementar, uma busca manual foi realizada nos três engenhos, visando identificar estudos de interesse deste trabalho e que não foram retornados na busca automática.

A lista final de estudos retornados foi analisada. Em um primeiro momento foram lidos o título e o resumo. Posteriormente, foi feita a leitura da introdução e conclusão dos estudos pré-selecionados. Nas duas etapas, quando um estudo atendia a pelo menos um dos critérios de exclusão ele era retirado do processo de análise. Os estudos considerados relevantes foram lidos na íntegra, sendo feita a extração e análise dos dados que respondessem às questões de pesquisa definidas.

\subsection{Extração e síntese dos dados}

Nesta fase, os seguintes dados foram extraídos dos estudos selecionados: título do artigo, autor(es), onde foi publicado (conferência / revista), ano de publicação, instituição do(s) autor(es), país da(s) instituição(ões), objetivo(s) da pesquisa, nível de ensino em que o estudo foi feito, número de estudantes envolvidos no estudo, faixa etária média dos estudantes envolvidos no estudo, tipos de recursos educacionais recomendados, técnica de recomendação utilizada, método de pesquisa, tipo de coleta de dados utilizado, principais resultados e limitações do estudo e sugestões do estudo 
VI Congresso Brasileiro de Informática na Educação (CBIE 2017)

Anais do XXVIII Simpósio Brasileiro de Informática na Educação (SBIE 2017)

para futuras pesquisas. Além disso, outros trechos dos artigos considerados importantes foram extraídos visando dar suporte às respostas das perguntas de pesquisa deste mapeamento.

\section{Resultados e discussões}

A busca resultou em 38 artigos completos, dos quais 22 foram selecionados para extração dos dados, considerando os critérios de inclusão e exclusão estabelecidos.. A Tabela 1 apresenta os resultados gerais dos processos de pré-seleção e inclusão dos artigos para o SBIE, WIE e RBIE.

A lista com as referências completas dos estudos primários está disponível no link: http://bit.ly/2teUWzt

Tabela 1. Resultado geral das buscas para o SBIE, WIE e RBIE

\begin{tabular}{|l|l|l|l|}
\hline Fonte & \multicolumn{1}{|c|}{$\begin{array}{c}\text { No de Estudos } \\
\text { Retornados }\end{array}$} & \multicolumn{1}{|c|}{$\begin{array}{c}\text { No de Estudos } \\
\text { Analisados }\end{array}$} & \multicolumn{1}{c|}{$\begin{array}{c}\text { \% de Estudos } \\
\text { Analisados }\end{array}$} \\
\hline SBIE & 26 & 15 & $68,2 \%$ \\
\hline WIE & 0 & 0 & $0 \%$ \\
\hline RBIE & 12 & 7 & $31,8 \%$ \\
\hline Total & 38 & 22 & $100 \%$ \\
\hline
\end{tabular}

\subsection{Evolução temporal dos estudos}

O gráfico apresentado na Figura 1 apresenta a evolução histórica das publicações sobre SRE em veículos brasileiros de publicação sobre IE. É possível perceber que o SBIE, considerado o evento mais importante na área de IE no Brasil, teve maior número de publicações, sobretudo nos anos 2013 e 2015. Além disso, observa-se que a quantidade de artigos por ano, a partir de 2013 se estabilizou, demonstrando que o tema ainda é relevante. Como o WIE não teve estudos selecionados, ele não aparece no gráfico.

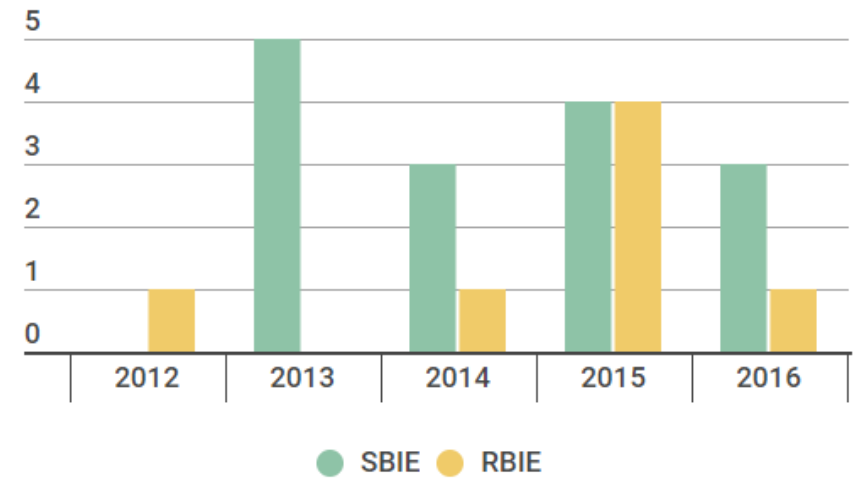

Figura 1. Evolução temporal dos estudos

\subsection{Instituições de pesquisa e distribuição geográfica}

$\mathrm{Na}$ Tabela 2 é apresentada a quantidade de artigos por instituições de pesquisa. Com exceção da Universidade Federal de Juiz de Fora (UFJF), a Universidade do Vale do Rio dos Sinos (UNISSINOS) e a Universidade Federal de Uberlândia (UFU) que 
VI Congresso Brasileiro de Informática na Educação (CBIE 2017)

Anais do XXVIII Simpósio Brasileiro de Informática na Educação (SBIE 2017)

tiveram mais de um trabalho, as demais instituições tiveram, cada uma, apenas 1 artigo incluído neste mapeamento.

Tabela 2. Instituições envolvidas nas pesquisas

\begin{tabular}{|l|l|l|l|}
\hline \multicolumn{1}{|c|}{ Instituição } & Quantidade de Artigos & \multicolumn{1}{|c|}{ Instituição } & $\begin{array}{c}\text { Quantidade de } \\
\text { Artigos }\end{array}$ \\
\hline UFAM/ IFAM/ UFRGS & 1 & IFMA/ UEMA/ SEDUC & 1 \\
\hline UFJF & 4 & UFSC & 1 \\
\hline UFCG/ UFAL & 1 & UNISSINOS & 3 \\
\hline UFPEL & 1 & UFU & 2 \\
\hline UFAM & 1 & PUCMG & 1 \\
\hline UFU/ IFSULDEMINAS & 1 & PPGCC - INE - UFSC & 1 \\
\hline PUCRS & 1 & $\begin{array}{l}\text { UFSCAR/ USP/ } \\
\text { FACENS }\end{array}$ & 1 \\
\hline UFSCAR & 1 & UFRJ & 1 \\
\hline
\end{tabular}

A distribuição geográfica das instituições que realizaram as pesquisas apresentadas nos artigos é mostrada na Figura 2. Aproximadamente 68,2\% das pesquisas apresentadas nos artigos foram realizadas por instituições localizadas nas regiões Sul e Sudeste do Brasil. Cerca de 4,5\% dos estudos estão na região Norte, enquanto que aproximadamente $27,3 \%$ dos artigos foram desenvolvidos de maneira colaborativa, contando com a participação de instituições de pesquisa situadas em dois ou mais locais. Neste último caso, 33,3\% dos trabalhos estão localizados nas regiões Norte e Nordeste, $50 \%$ dos trabalhos está localizado na região Sul e Sudeste e aproximadamente 16,7 \% dos trabalhos é uma colaboração de instituições das regiões Norte e Sul.

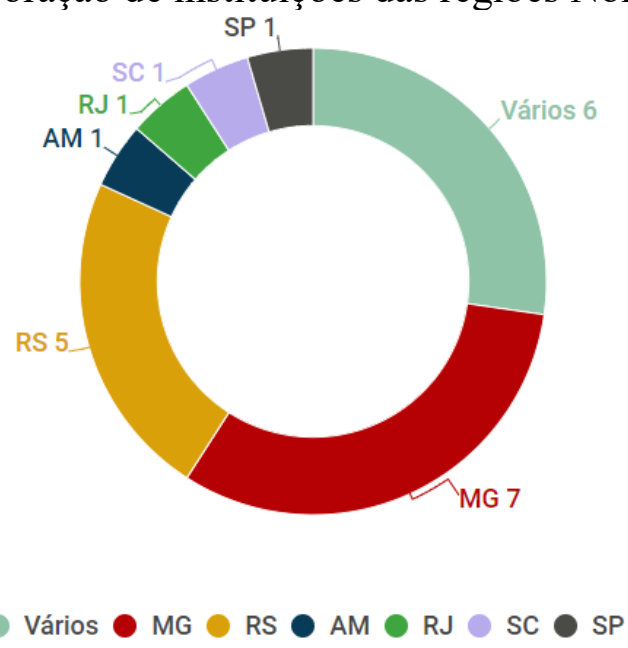

Figura 2. Estados das instituições envolvidas nas pesquisas 
VI Congresso Brasileiro de Informática na Educação (CBIE 2017)

Anais do XXVIII Simpósio Brasileiro de Informática na Educação (SBIE 2017)

\title{
4.3. Público-alvo
}

Em relação ao público-alvo das recomendações, os artigos foram classificados de acordo com o nível de escolaridade para os quais as pesquisas se destinam da seguinte forma: (i) Superior; (ii) Superior e Técnico; (iii) Superior e Profissionais; (iv) Técnico; (v) Médio; (vi) Profissionais em Geral; (vii) Não especifica. A distribuição dos estudos nessas categorias é apresentada na Figura 3.

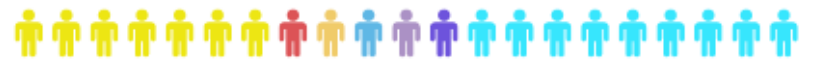

\author{
Superior $\bigcirc$ Superior e Técnico \\ Superior e Profissionais Técnico Médio \\ Profissionais, em geral $\bigcirc$ Não específica
}

Figura 3. Público-alvo dos estudos

É possível perceber, então, que a maioria dos trabalhos são focados em recomendar recursos educacionais para aqueles que estão no contexto de adquirir conhecimento, os estudantes. Também, mesmo em pequena quantidade, percebe-se que há iniciativas voltadas aqueles estão no papel de educadores e outros profissionais, evidenciando assim a escassez de trabalhos nesse contexto.

\subsection{Recursos educacionais recomendados}

Referente aos recursos educacionais recomendados ao usuário, os mesmos foram classificados da seguinte maneira: (i) Objetos de Aprendizagem (OA) que, por sua vez, podem ser vídeos, áudios, artigos, animações, textos, informações sobre estudantes, além de conteúdos do ambiente virtual analisado; (ii) Arquiteturas Pedagógicas (AP), que podem ser dinâmicas em grupo, ferramentas de apoio à cooperação, entre outros; (iii) Técnica de Aprendizagem (TA); (iv) Pessoas (P); (v) Predição de desempenho (PD), que, por sua vez, trata da análise de desempenho do estudante, classificando em zona de risco ou não; (vi) Quiz (Q); (vii) Jogos (J); (viii) Livros e Conteúdos Educacionais (LCE). Na Figura 4 é mostrada a distribuição dos artigos nessas categorias.

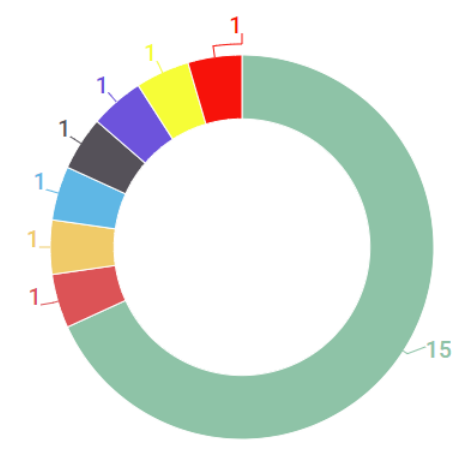

- $\mathrm{OA} \bullet \mathrm{AP} \odot \mathrm{TA} \odot \mathrm{P} \bullet \mathrm{PD} \bullet \mathrm{Q} \odot \mathrm{J} \bullet \mathrm{LCE}$

Figura 4. Distribuição dos trabalhos no que se refere aos recursos educacionais recomendados 
VI Congresso Brasileiro de Informática na Educação (CBIE 2017)

Anais do XXVIII Simpósio Brasileiro de Informática na Educação (SBIE 2017)

\subsection{Técnicas usadas}

Em se tratando das técnicas de recomendação mais utilizadas em SRE no Brasil, os trabalhos científicos pesquisados foram categorizados em: (i) Filtragem Híbrida (FH); (ii) Filtragem Colaborativa (FC); (iii) Filtragem Baseada em Contexto (FBCto); (iv) Filtragem Baseada em Perfil (FBP); (v) Filtragem Por Pares (FPP); (vi) Análise Diagnóstica (AD); (vii) Estilos de Aprendizagem (EA); (viii Filtragem Baseada em Metadados (FBM); e (ix) Filtragem Baseado em Conteúdos (FBC).

Com base na Figura 5, é possível perceber que a maior parte das pesquisas utiliza a técnica de recomendação colaborativa, representando cerca de $46,7 \%$ dos estudos. Representando $40 \%$ das pesquisas, técnicas de recomendação personalizadas tem surgido e obtido bons resultados no que compete às recomendações.

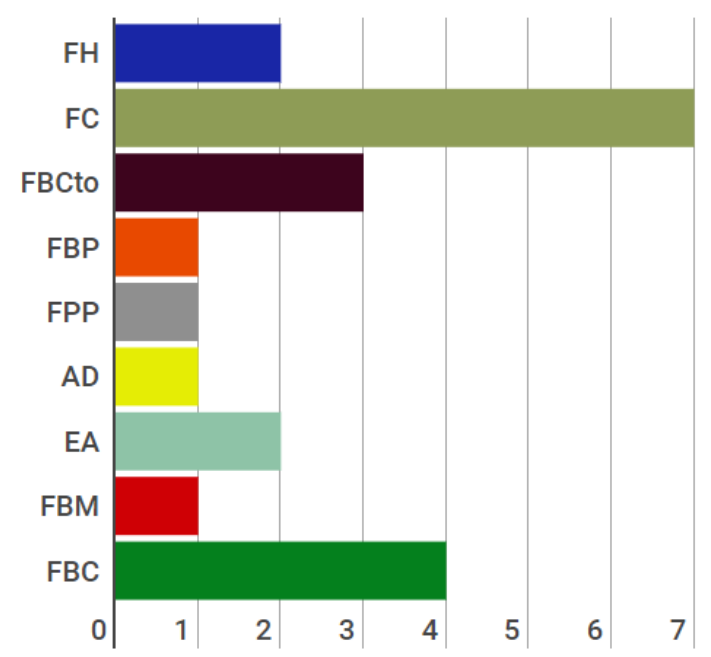

Figura 5. Distribuição dos trabalhos no que se refere às técnicas de recomendação

\subsection{Avaliação de SRE}

Sobre as avaliações de SRE realizadas nas pesquisas, elas foram caracterizadas do seguinte modo: (i) Estudo de Caso; (ii) Experimento; e (iii) Testes Funcionais.

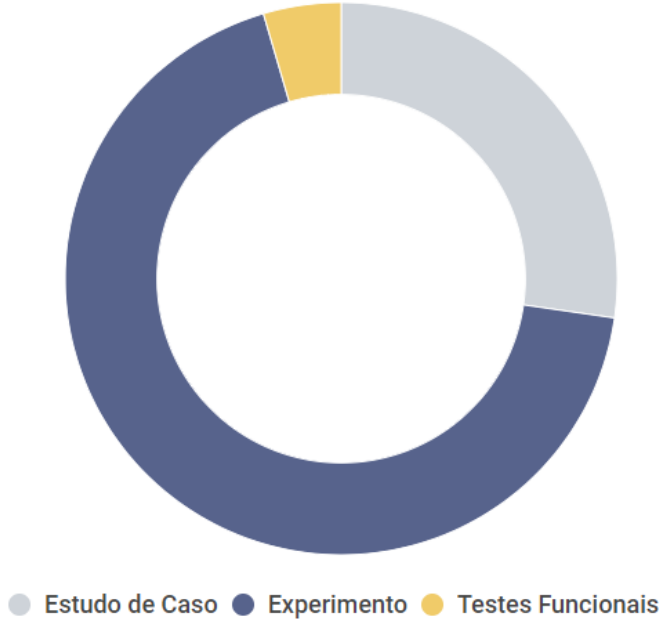

Figura 6. Distribuição das pesquisas em relação à avaliação de SRE 
É possível identificar na Figura 6 que, em 68,18\% das pesquisas, experimentos controlados foram realizados, porém em um dos casos os pesquisadores fizeram análise das métricas estatísticas Precisão e/ou Cobertura, dentre outras geralmente empregadas em SR. Ainda é possível perceber que estudos de casos foram utilizados com frequência, representando $27,27 \%$ das pesquisas. Também foram realizados testes funcionais, em 4,55\% dos trabalhos, utilizados no processo de validação e medição da qualidade do produto desenvolvido.

\section{Considerações finais}

Neste trabalho foram apresentados os resultados de um mapeamento sistemático sobre SRE que contou com a análise de 22 estudos publicados no período de 2012 a 2016 no SBIE e na RBIE. O WIE não retornou estudos sobre o tema. A partir da análise realizada verificou-se que o tema tem sido foco de diversas pesquisas no Brasil, tendo maior concentração nas regiões Sul e Sudeste. Foi possível identificar ainda que o público-alvo, foco dos estudos, são estudantes do ensino superior. Nesse sentido, percebendo o impacto que os SRE podem ter sobre a aprendizagem, investigações futuras podem considerar estudantes do ensino médio como público-alvo desses sistemas, como também formas de explorar seus benefícios no apoio à prática pedagógica docente.

Tendo em vista que OA são os recursos mais recomendados, outras pesquisas podem analisar de que maneira a colaboração pode ser explorada em benefício da aprendizagem, podendo focar na recomendação de pessoas, como alunos, em AVAs. Em relação às técnicas de recomendação utilizadas, foi possível perceber que os tipos tradicionais, como a Filtragem Colaborativa, ainda são bastante utilizados, porém algumas técnicas padronizadas têm surgido, tais como Filtragem Baseada Metadados e Filtragem Baseada em Conteúdos, alterando assim, o panorama das principais técnicas e trazendo bons resultados no que se diz respeito a recomendações de recursos educacionais. No que compete às avaliações realizadas nos SRE, se limitou a apenas três tipos de avaliação, sendo o experimento controlado observado na maior parte dos estudos analisados.

Em síntese, é possível concluir que este mapeamento sistemático permite aos pesquisadores ter um panorama sobre SRE no Brasil, comprimindo e facilitando o acesso a dados publicados das pesquisas na temática abordada. Seus resultados ainda apontam tendências de pesquisas e lacunas que poderão ser foco de futuras investigações.

Algumas limitações circundam este trabalho, tendo a mais comum das limitações nas revisões sistemáticas como principal. Esta limitação se refere à execução de todo processo, desde a pré-seleção dos artigos, até a extração dos dados, podendo estudos ou dados relevantes não terem sido considerados no processo de análise. Outra limitação está na quantidade de bases inseridas: apenas três foram selecionadas. Contudo, selecionou-se os três principais veículos de publicação nacional sobre IE no intuito de ter-se uma visão mais próxima da pesquisa sobre SRE no cenário nacional. Outra limitação se refere ao mecanismo de busca das bases analisadas, o qual não permite ao pesquisador construir uma string de busca avançada, fazendo-se necessário, neste trabalho, completar o processo com uma busca manual. 
VI Congresso Brasileiro de Informática na Educação (CBIE 2017)

Anais do XXVIII Simpósio Brasileiro de Informática na Educação (SBIE 2017)

Como trabalhos futuros pretende-se expandir este mapeamento englobando bases de dados internacionais, como ACM Digital Library, IEEE Xplore Digital Library, SCOPUS e ScienceDirect. Com a análise de desses estudos, será possível verificar se há divergências entre as pesquisas nacionais e internacionais sobre SRE.

\section{Referências}

Aguiar, J. J., Santos, S. I. N., Fechine, J. M., \& Costa, E. B. (2015). Avaliação de Sistemas de Recomendação Educacionais no Brasil: uma revisão sistemática da literatura. In XXVI Simpósio Brasileiro de Informática na Educação (SBIE), Maceió, AL.

Aguiar, J. J., Santos, S. I., Fechine, J. M., \& Costa, E. B. (2014). Um Mapeamento Sistemático sobre Iniciativas Brasileiras em Sistemas de Recomendação Educacionais. In XXV Simpósio Brasileiro de Informática na Educação (SBIE), Dourados, MS.

Associação Brasileira de Educação a Distância - ABED. Relatório Analítico da Aprendizagem a Distância no Brasil. 2015. Acesso em 3 de março de 2017. Disponível em: <https://goo.gl/F4XaGc>

Aureliano, V. C. O., \& Tedesco, P. C. D. A. R. (2012). Ensino-aprendizagem de Programação para Iniciantes: uma Revisão Sistemática da Literatura focada no SBIE e WIE. In Simpósio Brasileiro de Informática na Educação (SBIE), Rio de Janeiro, RJ.

Cazella, S. C., Reategui, E. B., Machado, M., \& Barbosa, J. L. V. (2009). Recomendação de objetos de aprendizagem empregando filtragem colaborativa e competências. In Simpósio Brasileiro de Informática na Educação (SBIE), Florianópolis, SC.

Costa, E., Aguiar, J., \& Magalhães, J. (2013). Sistemas de Recomendação de Recursos Educacionais: conceitos, técnicas e aplicações. In Jornada de Atualização em Informática na Educação (JAIE), Campinas, SP.

Kitchenham (2007). Guidelines for performing Systematic Literature Reviews in Software Engineering. In EBSE Technical Report. Acesso em 15 jul. 2017. Disponível em $<$ https://www.cs.auckland.ac.nz/ mria007/Sulayman/Systematic_reviews_5_8.pdf $>$

Magalhães, C. V., Santos, R. E., da Silva, F. Q., \& Gomes, A. S. (2013). Caracterizando a pesquisa em informática na educação no Brasil: um mapeamento sistemático das publicações do SBIE. In Simpósio Brasileiro de Informática na Educação (SBIE), Campinas, SP.

Pontes, W. L., França, R. M., Costa, A. P. M., \& Behar, P. (2014). Filtragens de Recomendação de Objetos de Aprendizagem: uma revisão sistemática do CBIE. In Simpósio Brasileiro de Informática na Educação (SBIE), Dourados, MS. 